\title{
Dynamics of localized magnetic inhomogeneities in the five-layer ferromagnetic structure
}

\author{
E.G. Ekomasov ${ }^{\dagger}$ A.M. Gumerov, R.V. Kudryavtsev \\ †EkomasovEG@gmail.com
}

Bashkir State University, Z. Validi St. 32, 450076, Ufa, Russia

\begin{abstract}
The paper considers the five-layer ferromagnetic structure, consisting of three identical wide layers separated by two thin layers with of the same the changed values of the anisotropy parameter. Anisotropy parameters are considered to be functions of the coordinate, directed perpendicular to the interface between the layers, i.e. we believe that the system has two magnetic «defects.» Has been studied the case of magnetic point defects described by a Dirac delta function with the parameters of the magnetic anisotropy different from the values of the magnetic anisotropy parameter in the rest of the magnet. With an approximate collective coordinate approach used previously to analyze the vibrations localized nonlinear magnetization waves at a single point defect theoretically studied the collective effect of two identical magnetic defects on the dynamics of coupled nonlinear magnetization waves. Has been studied the structure and dynamics of localized magnetic inhomogeneity of type four-kink multisoliton. For small amplitudes shown that oscillations of the magnetic multisoliton can be described as a system of two harmonic oscillators with elastic coupling type with the same natural frequency. In this case, the coupling coefficient can be varied, for example, by changing the distance between the defects. With increasing distance between defects, the coupling coefficient is reduced to zero, and the system is transformed into a system of differential equations for the uncoupled harmonic oscillators. In the case of reducing the distance between the defects is observed increase «stiffness» by effective communication between the oscillators.
\end{abstract}

Keywords: five-layer ferromagnetic structure, the dynamics of localized magnetic inhomogeneities, sine-Gordon equation.

\section{Динамика локализованных магнитных неоднородностей в пятислойной ферромагнитной структуре}

\author{
Екомасов $\dagger$ Е.Г., Гумеров А.М., Кудрявцев Р.В. \\ †EkomasovEG@gmail.com
}

Башкирский государственный университет, ул. З.Валиди 32, 450076, Уфа, Россия

В данной работе нами была рассмотрена пятислойная ферромагнитная структура, которая состоит из трёх широких одинаковых слоёв, разделённых двумя тонкими одинаковыми слоями с изменёнными значениями параметра магнитной анизотропии. Параметры магнитной анизотропии считаются функциями от координаты, направленной перпендикулярно границе раздела слоёв, т.е. мы считаем, что в системе имеются два магнитных «дефекта». В данной работе нами был исследован случай точечных магнитных дефектов, описываемых с помощью дельта функции Дирака, со значениями параметров магнитной анизотропии, отличающимися от значений параметров магнитной анизотропии в остальном магнетике. С помощью приближенного коллективно-координатного подхода, который использовался ранее для анализа колебаний локализованных нелинейных волн намагниченности на одиночном точечном дефекте, теоретически изучается коллективное влияние двух одинаковых магнитных дефектов на динамику связанных нелинейных волн намагниченности. В данной работе была исследована структура и динамика локализованной магнитной неоднородности типа четырехкинкового мультисолитона. Для случая малых амплитуд было показано, что колебания магнитного мультисолитона могут быть описаны системой двух гармонических осцилляторов со связью упругого типа с одинаковой собственной частотой. При этом, коэффициент связи можно изменять, например, с помощью изменения расстояния между дефектами. По мере увеличения расстояния между дефектами, коэффициент связи уменьшается до нуля, и система трансформируется в систему дифференциальных уравнений для несвязанных гармонических осцилляторов. В случае уменьшения расстояния между дефектами наблюдается увеличение «жёсткости» связи между эффективными осцилляторами.

Ключевые слова: пятислойный ферромагнетик, динамика локализованных магнитных неоднородностей, уравнение синус-Гордона. 


\section{1. Введение}

В последнее время широко исследуются многослойные магнитные структуры в связи с возможностью их практического применения [1]. Часто они представляют собой периодически чередующиеся слои двух материалов с различными физическими свойствами. В настоящее время изучается динамика спиновых волн и магнитных неоднородностей, распространяющихся в таких системах как вдоль, так и перпендикулярно границам раздела слоёв. Во втором случае, часто используются одномерные модели $[2,3]$. Отметим, что зачастую именно изучение одномерных моделей позволяет понять влияние тех или иных магнитных параметров на рассматриваемый процесс (см., например, [4-5]).

При исследовании динамики линейных и нелинейных волн намагниченности, распространяющихся перпендикулярно слоям, существует два подхода. В первом из них, используемом часто для изучения динамики спиновых волн, для описания динамики намагниченности в слое рассматривается уравнение Ландау-Лифшица с постоянными параметрами материала, а на границе слоёв требуется выполнение определённых граничных условий [6]. Во втором подходе, наличие слоёв, отличающихся друг от друга значением одного или нескольких магнитных параметров, учитывается пространственной модуляцией магнитных параметров материала (см., например, $[7,8])$.

Влияние локальной и периодической одномерной пространственной модуляции магнитных параметров материала (ПММП) на характер распространения, спектр и затухание спиновых волн, и на высокочастотные свойства, изучено достаточно хорошо (см., например, [9]). При определённых условиях, изучение одномерной динамики магнитных неоднородностей приводит к интересной и с математической точки зрения задаче нахождения решения уравнения типа синус-Гордона с переменными коэффициентами, имеющего важное значение для многих областей современной физики $[1,10]$. В связи со сложностью задачи, исследователями рассматривалась, как правило, модуляция лишь отдельных параметров магнитной системы. Часто учитывалась, например, модуляция магнитной анизотропии для случая двух-, трёх- и пятислойного магнетика, причём задачи изучались как аналитическими, так и численными методами, как для точечных, так и для протяжённых дефектов $[2,11,12]$. В настоящей работе проведено аналитическое исследование динамики локализованных магнитных неоднородностей мультисолитонного типа, возбуждаемых на двух одинаковых точечных магнитных дефектах.

\section{2. Основные уравнения и результаты}

Рассмотрим пятислойную ферромагнитную структуру, состоящую из трёх широких одинаковых слоёв, разделённых двумя тонкими одинаковыми слоями с изменёнными значениями параметра анизотропии. Параметры анизотропии считаем теперь функциями от координаты $x$, направленной перпендикулярно границе раздела слоёв, т.е. в системе имеются два магнитных дефекта. Будем далее изучать динамику расположенных в плоскости $y z$ локализованных магнитных неоднородностей. Обычно, при решении динамических задач, удобно перейти к сферическим координатам вектора намагниченности $\mathbf{M}(\cos \varphi \sin \theta, \sin \varphi, \cos \varphi \cos \theta)$, где $0 \leq \theta \leq 2 \pi-$ угол в плоскости $y z$ между направлением вектора магнитного момента и осью лёгкого намагничивания (ось $O z),-\pi / 2<\varphi<\pi / 2-$ угол, описывающий выход $\mathbf{M}$ из плоскости доменной границы (ДГ). Учитывая в плотности энергии магнетика обменное взаимодействие и анизотропию, и считая $\varphi \ll 1$ [1], уравнение движения для намагниченности в угловых переменных можно представить в следующем обезразмеренном виде [12]:

$$
u_{t t}-u_{x x}+\sin u=[\varepsilon \delta(x)+\varepsilon \delta(x-d)] \sin u,
$$

где $u=2 \theta$, слагаемое $\varepsilon \delta(x)$ моделирует пространственную модуляцию магнитной анизотропии в виде точечного дефекта, $\delta(x)$ - дельта-функция Дирака, $\varepsilon-$ константа, $d$ - расстояние между дефектами. Уравнение (1) - модифицированное уравнение синус-Гордона (МУСГ). Заметим так же, что уравнение вида (1) можно получить и для случая двухподрешёточных ферримагнетиков и слабых ферромагнетиков.

Рассмотрим структуру и динамику локализованных нелинейных волн намагниченности, возбуждаемых, например, в результате рассеяния ДГ на точечных дефектах. Будем считать, что $\varepsilon<1$, а слагаемые в правой части уравнения (1) малы. Применим приближенный коллективно-координатный подход, использованный ранее для анализа колебаний локализованных нелинейных волн намагниченности на одиночном точечном дефекте [12]. Учитываем наличие локализованных волн намагниченности в области дефекта (или примесных мод) с помощью введения двух коллективных переменных $-a_{1}=a_{1}(t)$ и $a_{2}=a_{2}(t)-$ являющихся амплитудами этих волн. Выражение для примесных мод будем брать в виде, аналогичном использованному ранее для случая одиночного дефекта [10]:

$$
\begin{gathered}
u_{1}=a_{1}(t) \exp (-\varepsilon|x| / 2), \\
u_{2}=a_{2}(t) \exp (-\varepsilon|x-d| / 2) .
\end{gathered}
$$

В приближении малых колебаний, считаем, что

$$
a_{i}(t)=a_{i 0} \cos \left(\Omega_{\text {single }} t+\theta_{0}\right),
$$

где $\theta_{0}$ - начальная фаза. Решая (1) для случая одиночного дефекта, можно получить для частоты примесной моды следующее выражение:

$$
\Omega_{\text {single }}=\sqrt{1-\varepsilon^{2} / 4} .
$$

Общее решение задачи $-u_{\text {ansatz }}-$ будем искать в виде: $u_{\text {ansatz }}=u_{1}+u_{2}=a_{1}(t) \exp (-\varepsilon|x| / 2)+$

$$
+a_{2}(t) \exp (-\varepsilon|x-d| / 2) \text {. }
$$

Для нахождения аналитического решения считаем, что $a_{1}(t)$ и $a_{2}(t)$ достаточно малы (являются величиной порядка малого $\varepsilon$ ). В рамках рассматриваемого приближения можно считать, что $u_{\text {ansatz }}<1$. Тогда нелинейный член в лагранжиане, приводящем к уравнению (1) (см. $[7,10])$, можно разложить в ряд Тейлора до членов второго порядка по $\varepsilon$ :

$$
\cos u-1 \approx-u^{2} / 2 \text {. }
$$

Подстановка (5) в лагранжиан, приводящий к уравнению (1), с учётом приближений, приводит после интегрирования к новому эффективному лагранжиану, за- 
висящему уже от новых переменных $a_{1}$ и $a_{2}$. Уравнения движения для $a_{1}(t)$ и $a_{2}(t)$ можно получить, подставив полученный эффективный лагранжиан в систему уравнений Лагранжа второго рода:

$$
\begin{aligned}
& \ddot{a}_{1}(t)+a_{1}(t) \Omega_{0}^{2}=F\left(a_{2}(t)-a_{1}(t)\right) \\
& \ddot{a}_{2}(t)+a_{2}(t) \Omega_{0}^{2}=-F\left(a_{2}(t)-a_{1}(t)\right)
\end{aligned}
$$

где

$$
\begin{gathered}
F=F(\varepsilon, d)=\frac{\varepsilon^{2}\left(2-(2+\varepsilon d) e^{-\varepsilon d}\right) e^{-\varepsilon d / 2}}{4-(2+\varepsilon d)^{2} e^{-\varepsilon d}} \\
\Omega_{0}^{2}=\Omega_{0}^{2}(\varepsilon, d)=1-\frac{\varepsilon^{2}}{4}-\frac{\varepsilon^{2}\left(1+e^{-\varepsilon d / 2}\right) e^{-\varepsilon d / 2}}{2+(2+\varepsilon d) e^{-\varepsilon d / 2}}
\end{gathered}
$$

Система уравнений (7) - система обыкновенных дифференциальных уравнений второго порядка. Из (7) следует, что колебания системы связанных локализованных волн намагниченности бризерного типа в приближении малых колебаний можно описать системой связанных эффективных гармонических осцилляторов с одинаковой собственной частотой $\Omega_{0}(\varepsilon, d)$. На каждый из них действует внешняя сила $\left(a_{2}(t)-a_{1}(t)\right) F(\varepsilon, d)$ (упругого типа) со стороны «соседнего» осциллятоpa. При этом, коэффициент связи $F(\varepsilon, d)$ можно изменять, например, с помощью изменения параметра $d-$ расстояния между примесями. Проанализируем поведение функций (8) и (9) в предельных случаях (см. рис. 1). С увеличением параметра $d$, коэффициент связи уменьшается до нуля, и (7) переходит в систему уравнений для несвязанных гармонических осцилляторов. При этом, $\Omega_{0}(\varepsilon, d)$ переходит в выражение для частоты, полученной для случая одиночного дефекта $\Omega_{\text {single }}=\left(1-\varepsilon^{2} / 4\right)^{1 / 2}$. В этом случае осцилляторы колеблются независимо друг от друга на собственной частоте примесной моды, возбуждаемой на одиночном дефекте. В случае $d \rightarrow 0$ данная зависимость $F(\varepsilon, d)$ начинает сильно возрастать, что соответствует увеличению «жёсткости» связи между эффективными осцилляторами. При этом, величина $\Omega_{0}(\varepsilon, d=0)$ имеет конечное значение, соответствующее «удвоенному» эффективному осциллятору.

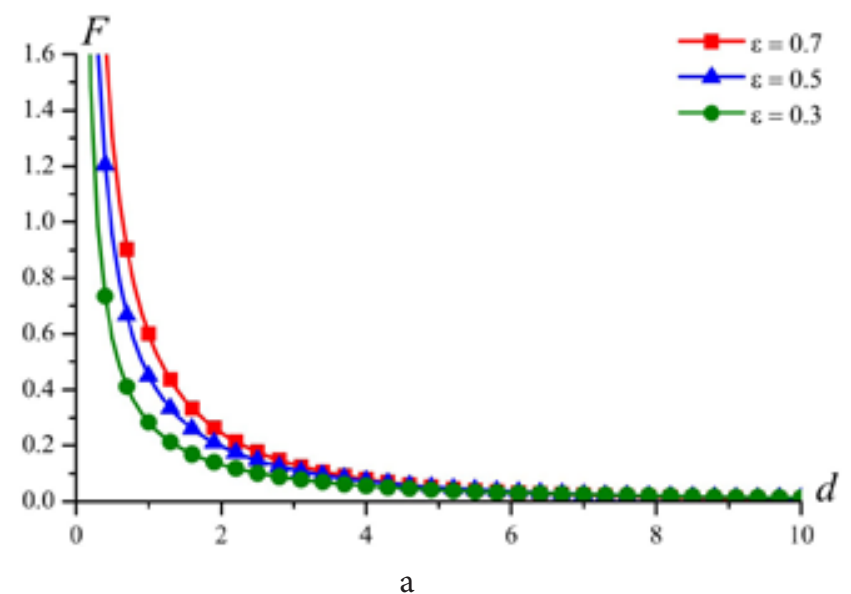

\section{Литература/References}

1. A. Hubert, R. Schafer. Magnetic domains. SpringerVerlag, Hedelberg, Berlin. (1998) 696 p.

2. A.B. Borisov, V.V. Kiselev. Nonlinear waves, solitons and localized structures in magnetic materials. T.1. Quasi-one-dimensional magnetic solitons. UB RAS, Ekaterinburg. (2009) 512 p. (in Russian) [А.Б. Борисов, В.В. Киселёв. Нелинейные волны, солитоны и локализованные структуры в магнетиках. Т.1. Квазиодномерные магнитные солитоны. УрО РАН, Екатеринбург. (2009) 512 с.

3. E. Della Torre, C.M. Perlov. J. Appl. Phys. 69, 4596 (1991).

4. V.N. Nazarov, R.R. Shafeev, M.A. Shamsutdinov, I.Yu Lomakina. Phys. Solid State. 54, 298 (2012). (in Russian) [В.Н. Назаров, Р.Р. Шафеев, М.А. Шамсутдинов, И.Ю. Ломакина. ФТТ. 54(2), 282 (2012).]

5. V.V. Kiselev, A.A. Rascovalov. Chaos, Solitons \& Fractals. 45, 1551 (2012).

6. V.V. Kruglyak, A.N. Kuchko, V.I. Finokhin. Phys. Solid State. 46, 867 (2004). (in Russian) [В.В. Кругляк, А.Н. Кучко, В.И. Финохин. ФТТ. 46(5), 842 (2004).]

7. E. G. Ekomasov, A. M. Gumerov, R. R. Murtazin, R. V. Kudryavtsev, A. E. Ekomasov, N. N. Abakumova. Solid state phenomena. 233-234, 51-54 (2015).

8. E.G. Ekomasov, R.R. Murtazin, V.N. Nazarov. Journal of Magnetism and Magnetic Materials. 385, 217 (2015).

9. V.A. Ignatchenko, Yu.I. Mankov, A.A. Maradudin. Phys. Rev. B. 62(3), 2181 (2000).

10. J. Cuevas-Maraver, P. G. Kevrekidis, F. Williams (Eds.). The Sine-Gordon Model and Its Applications: From Pendula and Josephson Junctions to Gravity and Highenergy Physics, volume 10. Springer. (2014) 263 p.

11. E.G. Ekomasov, R.R. Murtazin, O.B. Bogomazova, A.M. Gumerov. JMMM. 339, 133 (2013).

12. E. G. Ekomasov, A. M. Gumerov. Letters on materials. 4, 237 (2014). (in Russian) [Е.Г. Екомасов, А.М. Гумеров. Письма о материалах. 4(4), 237 (2014).]

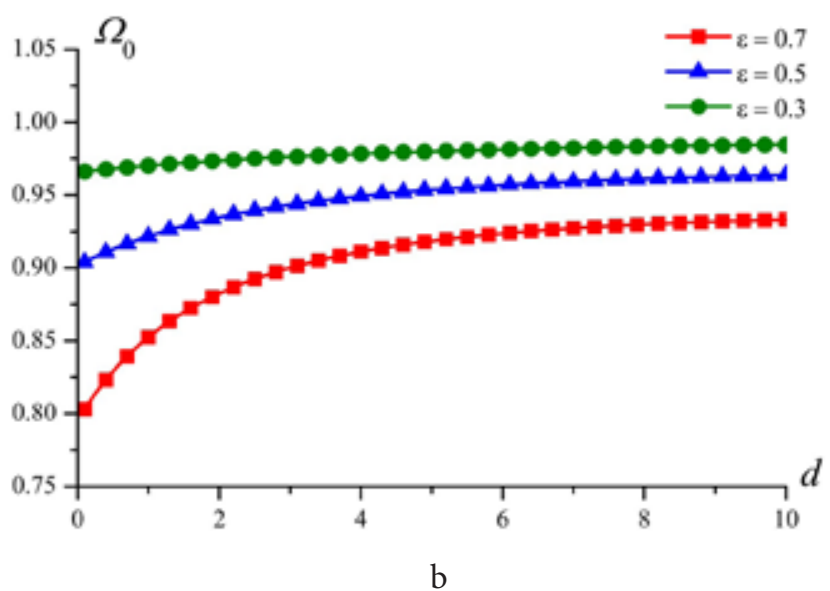

Pис. 1. (Color online) Зависимость от расстояния между дефектами $d$ : (а) коэффициента связи $F(\varepsilon, d)$; (b) собственной частоты колебаний $\Omega_{0}(\varepsilon, d)$.

Fig. 1. (Color online) Dependency on the distance between the defects $d$ : (a) coupling factor $F(\varepsilon, d)$; (b) the natural frequency of oscillation $\Omega_{0}(\varepsilon, d)$. 